\title{
The correct name for Matelea chacoensis Goyder (Apocynaceae: Asclepiadoideae)
}

\section{David J. Goyder ${ }^{1}$}

Summary. Matelea chacoensis Goyder was proposed as a replacement name for the illegitimate Gonolobus hirtus Griseb., a later homonym of G. hirtus (L.) R. Br. ex Schultes. However, the synonym Pseudibatia hirta Malme, which may be considered a legitimate replacement name, makes an earlier epithet available. The correct combination under Matelea is therefore M. hirta (Malme) Goyder.

Key Words. Asclepiadaceae, Bolivia, nomenclature, Pseudibatia.

Goyder (2006: 32) proposed Matelea chacoensis Goyder as a replacement name for the illegitimate Gonolobus hirtus Griseb. (Grisebach 1879: 234), a later homonym of G. hirtus (L.) R. Br. ex Schultes (1820: 64). The name was adopted in the recent Southern Cone and Bolivian Checklists (Ezcurra et al. 2008: 1116; Fuentes \& Morales 2014: 244). However, Pseudibatia hirta Malme (1905: 14), which may be considered a legitimate replacement name (McNeill et al. 2012: Art. 58.1), was included in synonymy (as $P$. hirta (Griseb.) Malme). The epithet is available in Matelea and takes priority over M. chacoensis - the formal combination is made below.

Matelea hirta (Malme) Goyder, comb. nov.

http://www.ipni.org/urn:lsid:ipni.org:names:77152988-1

Basionym: Pseudibatia hirta Malme, Arkiv Bot. 4 (14): 14 (1905). Based on Gonolobus hirtus Griseb. (Grisebach 1879: 234), non G. hirtus (L.) R. Br. ex Schultes (1820: 64). Type: Argentina, Salta, "ad fl. Juramento", Feb. 1873, Lorentz $\mathcal{E}$ Hieronymus 316 (holotype GOET; isotype LIL).

Synonym: Matelea chacoensis Goyder (2006: 32), nom. superfl.

\section{Acknowledgements}

I am grateful to both Rafaël Govaerts (RBG Kew) and Cecilia Ezcurra (BCRU, Bariloche, Argentina) for bringing this matter to my attention.

\section{References}

Ezcurra, C., Fontella Pereira, J. \& Kinoshita, L. S. (2008). Apocynaceae. In: F. O. Zuloaga, O. Morrone \& M. J. Belgrano (eds), Catálogo de las Plantas Vasculares del Cono Sur: (Argentina, Sur de Brasil, Chile, Paraguay y Uruguay). Vol. 2, Dicotyledoneae: Acanthaceae - Fabaceae (Abarema - Schizolobium). Pp. 1090 - 1143. Missouri Botanical Garden Press, St Louis.

Fuentes, A. \& Morales, J. F. (2014). Apocynaceae. In: P. M. Jorgensen, M. H. Nee \& S. G. Beck (eds), Catálago de las plantas de Bolivia, pp. 232 - 255. Missouri Botanical Garden Press, St Louis.

Goyder, D. J. (2006). Rojasia reinstated and six new names and combinations in Matelea (Apocynaceae: Asclepiadoideae). Kew Bull. 61: 31 - 33.

Grisebach, A. (1879). Asclepiadeae. In: Symbolae at floram argentinam, pp. 225 - 235. Dieterich'sche Verlags-Buchhandlung, Göttingen.

Malme, G. O. A. (1905). Adnotationes de nonnullis Asclepiadaceis autroamericanis. Arkiv Bot. 4(14): 1 - 19.

McNeill, J., Barrie, F. R., Buck, W. R., Demoulin, V., Greuter, W., Hawksworth, D. L., Herendeen, P. S., Knapp, S., Marhold, K., Prado, J., Prud'homme van Reine, W. F., Smith, G. F., Wiersema, J. H. \& Turland, N. J. (2012). International Code of Nomenclature for algae, fungi, and plants (Melbourne Code). Regnum Veg. 154: i - xxx; $1-208$.

Schultes, R. A. (1820). Classis V. Pentándria Digynia. 1084. Gonolobus. Systema vegetabilium: secundum classes, ordines, genera, species, ed. 15, 6: 59 - 68 . Sumtibus J. G. Cottae, Stuttgardtiae.

Accepted for publication 9 February 2016. Published online 26 April 2016

1 Herbarium, Royal Botanic Gardens Kew, Richmond, Surrey, TW9 3AE, UK. e-mail: d.goyder@kew.org 